\title{
A INFLUÊNCIA DA CAPACIDADE DE MEMÓRIA DE TRABALHO NA GERAÇÃO DE INFERÊNCIAS E NA COMPREENSÃO LEITORA
}

\author{
Deise Caldart ROSCIOLI* \\ Lêda Maria Braga TOMITCH**
}

- RESUMO: Esta pesquisa teve como objetivo investigar se existe uma relação entre a Capacidade de Memória de Trabalho de estudantes do Ensino Médio Técnico, a geração de inferências e a compreensão leitora em L2. Um grupo de 36 alunos do terceiro ano do Curso Técnico em Agropecuária Integrado ao Ensino Médio do Instituto Federal do Rio Grande do Sul (IFRS) - Campus Sertão participou deste estudo. Os participantes eram falantes pré-intermediários de inglês como $\mathrm{L}^{2}{ }^{1}$. Os instrumentos utilizados neste estudo incluíram o Teste de Capacidade de Leitura (Reading Span Test); dois textos, sendo um narrativo e um expositivo; o Protocolo Pausa; e dois conjuntos de perguntas de compreensão (um para cada texto). Os dados coletados através do Teste de Capacidade de Leitura (Reading Span Test), as inferências geradas durante a leitura (categorizadas de acordo com o Modelo de Categorização de Inferências proposto por Narvaez, Broek e Ruiz (1999), bem como as respostas dos participantes às perguntas de compreensão foram analisadas e avaliadas tanto quantitativa quanto qualitativamente, sendo que os principais resultados mostram que a Capacidade de Memória de Trabalho se correlaciona positivamente com a compreensão leitora e também com inferências explicativas, que estão diretamente ligadas à compreensão.

- PALAVRAS-CHAVE: compreensão leitora; capacidade da memória de trabalho; geração de inferências.

\section{Introdução}

A influência da capacidade de memória de trabalho (CMT) dos indivíduos na realização de tarefas cognitivas complexas tem sido amplamente pesquisada. Também

\footnotetext{
Instituto Federal de Educação, Ciência e Tecnologia do Rio Grande do Sul (IFRS), Sertão - RS - Brasil. deise. roscioli@sertao.ifrs.edu.br. ORCID: 0000-0002-2827-5212

** Universidade Federal de Santa Catarina (UFSC), Florianópolis - SC - Brasil. Departamento de Língua e Literatura Estrangeira. leda@cce.ufsc.br. ORCID: 0000-0003-4183-8072

1 No presente estudo os termos segunda língua/ L2 e língua estrangeira/LE estão sendo utilizados de forma intercambiável.
} 
houve um aumento considerável no número de estudos relacionados ao processo de geração de inferências, principalmente nos últimos trinta anos. No entanto, estudos que investigam a relação entre a CMT dos indivíduos e a geração de inferências ainda necessitam de mais investigações, especialmente porque a maioria das pesquisas sobre CMT e geração de inferências tem sido realizada em L1, e não em segunda língua (L2) ou línguas estrangeiras (LE). Da mesma forma, embora os pesquisadores tenham demonstrado interesse em determinar as circunstâncias em que algumas inferências específicas são geradas, bem como os efeitos das características individuais dos leitores na atividade inferencial (NARVAEZ; BROEK; RUIZ, 1999), este último não tem recebido a mesma atenção. Diante dessa perspectiva, a necessidade de preencher tais lacunas é a motivação deste estudo, que faz parte de uma pesquisa de doutorado e tem como objetivo investigar como a CMT de alunos afeta sua geração de inferências e compreensão leitora, mais especificamente em relação aos alunos brasileiros matriculados em um Curso Técnico de Ensino Médio.

\section{Compreensão Leitora e Geração de Inferências}

No presente estudo e em consonância com grande parte da literatura da área, a leitura é entendida como um processo de construção de sentido, que é o resultado da interação entre texto e leitor (DAVIES, 1995; ESKEY, 1998; KINTSCH; DIJK, 1978; RUMELHART, 1977; TOMITCH, 2003; URQUHART; WEIR, 1998). A leitura aqui também é vista como interativa no sentido proposto por Rumelhart (1981), ou seja, processos de nível superior (por exemplo, o uso de conhecimento prévio na geração de inferência) podem interagir com processos de nível inferior (por exemplo, decodificação) a fim de alcançar a compreensão.

Para os fins do presente estudo, o termo inferência refere-se à "qualquer informação sobre eventos, relações e assim por diante que o leitor acrescente à informação que está explicitamente apresentada no texto"2 (BROEK; RISDEN 1995, p.353, tradução nossa). Koda (2008) complementa essa definição afirmando que a geração de inferência é uma parte do processo de leitura e é crucial para a 'construção do significado do texto'. Ao ler a frase 'João caiu no chão. Ele ficou uma semana inteira em casa' (CALDART, 2012), a maioria dos leitores consegue inferir que João teve que ficar em casa porque se machucou ao cair no chão, e ainda que seu acidente foi relativamente grave, devido ao período em que teve que ficar em casa para melhorar. Baretta (2008, p.138, nossa tradução) afirma que a capacidade de gerar inferências é "um processo cognitivo construtivo no qual o leitor se esforça para chegar ao significado e expande o conhecimento formulando e avaliando hipóteses sobre as informações do texto". ${ }^{3}$ Conforme afirmado por Dijk

\footnotetext{
No original "any information about events, relations, and so on that the reader adds to the information that is explicitly presented in the text." (BROEK; RISDEN 1995, p.353).

3 No original "a constructive cognitive process in which the reader strives for meaning and expands knowledge by formulating and evaluating hypotheses about the information in the text." (BARETTA, 2008, p.138).
} 
e Kintsch (1983) para que a compreensão de leitura ocorra, o leitor precisa construir uma representação mental adequada da mensagem, conectando as informações tanto no nível local (microestrutura), quanto global (macroestrutura). Portanto, a construção de sentido está ligada às inferências geradas pelos leitores, desde que essas inferências forneçam as conexões que integram as informações textuais, ajudando-os a construir um modelo mental sólido, que pode levar a uma melhor compreensão, retenção e recordação das informações previamente lidas (GERBER; TOMITCH, 2008). Como os textos são compostos de informações isoladas, a capacidade de fazer inferências é fundamental para que ocorra a compreensão leitora. No processo de geração de inferências, o leitor conta com sua memória das frases anteriores, bem como com seu conhecimento prévio relacionado ao conteúdo do texto, a fim de estabelecer uma relação entre as frases que estão sendo lidas e as anteriores (BROEK; ROHLEDER; NARVAEZ, 1994; YEARI; BROEK, 2015).

\section{Categorizações de inferências}

Os pesquisadores interessados na compreensão do discurso têm desenvolvido taxonomias de tipos de inferência, acompanhadas por uma tentativa de definir seus papéis na compreensão (GRAESSER; SINGER; TRABASSO, 1994; BROEK; ROHLEDER; NARVAEZ, 1994; NARVAEZ; BROEK; RUIZ, 1999, dentre outros). Para os propósitos deste estudo será adotado o Modelo de Categorização de Inferências proposto por Narvaez, Broek e Ruiz (1999), visto que este tem sido amplamente utilizado na literatura relacionada à leitura e geração de inferência (TRABASSO; SUH, 1993; ZWAAN; BROWN, 1996; TRABASSO; MAGLIANO, 1996; NARVAEZ; BROEK; RUIZ, 1999; MAGLIANO; GRAESSER; TRABASSO, 1999; LINDERHOLM, 2002), e parece abranger a maioria dos enunciados dos participantes durante um Protocolo de Pausa, conforme observado em Caldart (2012).

O Modelo de Categorização de Inferências proposto por Narvaez, Broek e Ruiz (1999) é apresentado na Tabela 1: 
Tabela 1 - Modelo de Categorização de Inferências

proposto por Narvaez; Broek e Ruiz (1999)

\begin{tabular}{|c|c|}
\hline Tipo de Inferência & Características \\
\hline Explicações & $\begin{array}{l}\text { Estão relacionadas aos motivos pelos quais algo acontece e incluem } \\
\text { explicações baseadas em conhecimentos prévios ("Acho que essa é } \\
\text { a causa da era do gelo") e explicações baseadas no texto ("Deve ser } \\
\text { isso o que eles querem dizer com cinzas"); }\end{array}$ \\
\hline Associações & $\begin{array}{l}\text { Fornecem informações sobre características e funções de pessoas, } \\
\text { objetos e eventos no texto, incluindo associações prévias ("Isso me } \\
\text { lembra de um show de planetário que vi") e associações baseadas } \\
\text { no texto ("Ok, isso é no spa"); }\end{array}$ \\
\hline Predições & $\begin{array}{l}\text { Referem-se às inferências sobre as consequências futuras de um } \\
\text { evento específico ("Tudo bem, os gases os levarão ao objeto real"); }\end{array}$ \\
\hline Avaliações & $\begin{array}{l}\text { Estão relacionadas aos comentários sobre o conteúdo do texto } \\
\text { ("Acho que é uma afirmação muito forte"), a redação do texto ("Essa } \\
\text { frase foi difícil de dizer") ou o estado do leitor ("Estou meio que } \\
\text { perdendo a noção aqui, me distraindo"); }\end{array}$ \\
\hline $\begin{array}{l}\text { Quebras de Coerência } \\
\text { Baseadas no Texto: }\end{array}$ & $\begin{array}{l}\text { Referem-se a afirmações sobre a coerência do conteúdo do texto } \\
\text { ("Isso não faz sentido"); }\end{array}$ \\
\hline $\begin{array}{l}\text { Quebras de Coerência } \\
\text { Baseadas no } \\
\text { Conhecimento: }\end{array}$ & $\begin{array}{l}\text { Incluem afirmações sobre a incapacidade de compreensão dos } \\
\text { leitores como resultado da falta de conhecimento ou experiência } \\
\text { ("É meio difícil de imaginar, quero dizer, no espaço"); }\end{array}$ \\
\hline Repetições & Referem-se às repetições de palavras ou frases no texto. \\
\hline
\end{tabular}

Fonte: Narvaez, Broek e Ruiz (1999, p.490, tradução nossa).

A geração de inferências auxilia a compreensão leitora, conectando as informações apresentadas no texto aos conhecimentos prévios dos leitores. Se os leitores não conseguirem fazer essas conexões, eles podem ter dificuldades para lembrar e até mesmo compreender o texto (BARETTA, 2008; HORIBA, 2000; LINDERHOLM; BROEK, 2002; TRABASSO; SUH, 1993). A fim de que os leitores possam fazer as inferências necessárias ao ler um texto, eles devem ser capazes de conectar as informações relevantes em sua memória de trabalho e também acessar o conhecimento prévio, se o conteúdo do texto não for suficiente. Portanto, parece legítimo levantar a hipótese de que o sucesso da realização de inferências depende da capacidade de memória de trabalho do leitor, tópico a ser discutido na seção a seguir.

\section{Capacidade da Memória de Trabalho}

O papel desempenhado pela memória de trabalho (MT) na cognição humana é inegável (DANEMAN; CARPENTER, 1980; BADDELEY, 1990; JUST; CARPENTER, 
1992; TOMITCH, 2003; entre outros); desde a leitura de um livro até a solução de problemas matemáticos complexos é necessário que não apenas as informações apresentadas sejam retidas temporariamente, mas que essas informações sejam processadas ao mesmo tempo, e é aí que entra a memória de trabalho.

Memória de trabalho é um conceito originalmente proposto por Baddeley e Hitch, em 1974, e tem sido usado desde então para se referir à capacidade dos indivíduos de não apenas armazenar informações, como é o caso da memória de curto prazo, mas também processar as porções de informações que vêm do texto. A memória de trabalho é definida como "uma arena de computação" (JUST; CARPENTER, 1992), onde as funções de processamento e armazenamento competem pela capacidade do sistema (BADDELEY; HITCH, 1974; DANEMAN; CARPENTER, 1980, 1983; JUST; CARPENTER, 1992). É um sistema multicomponente responsável pelo armazenamento e manipulação de informações durante a execução de tarefas consideradas cognitivamente complexas, como aprendizagem, compreensão e raciocínio (BADDELEY; HITCH, 1974; DANEMAN; CARPENTER, 1980; BADDELEY; LOGIE, 1999).

A memória de trabalho está, sem dúvida, envolvida na aprendizagem de uma segunda língua. Diversas pesquisas têm mostrado que a capacidade de memória de trabalho (CMT) está intimamente ligada à capacidade de realizar tarefas cognitivas e relacionadas à linguagem (ver, por exemplo, WALTER, 2006; LEESER, 2007; JUFFS; HARRINGTON, 2011; YEARI, 2017).

Afirmar que a capacidade de memória de trabalho dos indivíduos pode prever seu desempenho em uma tarefa implica afirmar que a MT é limitada. É importante ressaltar que embora possa ser difícil se chegar a um consenso sobre o que limita a MT, tanto teorias anteriores quanto mais recentes concordam que ela é transiente e que sua capacidade é limitada (TOMITCH, 2003). As teorias anteriores afirmam que a limitação reside no número de itens que podem ser armazenados de uma só vez. Teorias mais recentes acreditam que o que é limitado na memória de trabalho são os recursos relacionados à atenção que estão disponíveis para armazenamento e processamento de informações. Ashcraft (1994) apresenta uma desambiguação muito clara dos dois construtos (memória de curto prazo e memória de trabalho) e sua capacidade limitada; segundo ele, a memória de curto prazo, como o nome sugere, é muito curta, o que significa que não dura muito. Partindo do mesmo raciocínio, a memória de trabalho implica, pelo uso do verbo ativo 'trabalhar', que ela é dinâmica e que toda atividade mental acontece ali. A limitação no caso da MT diz respeito a "quanto trabalho pode ser feito de uma vez, quanta capacidade de memória de trabalho existe para ser compartilhada entre vários processos simultâneos" (ASHCRAFT, 1994, p.146, tradução nossa) $)^{4}$.

O modelo original proposto por Baddeley e seus colegas (BADDELEY; HITCH, 1974, 1994; BADDELEY, 1992; BADDELEY; LOGIE, 1999) é um modelo

\footnotetext{
4 No original "how much work can be done at one time, how much working memory capacity there is to share among several simultaneous processes." (ASHCRAFT, 1994, p.146).
} 
multicomponente de memória de trabalho e é composto por um sistema de controle de capacidade de atenção limitada: o executivo central, que é auxiliado por dois sistemas 'escravos': o circuito fonológico, que processa a informação verbal e fonológica; e a tábua de rascunho visual-espacial, que lida com informações visuais e espaciais. Posteriormente, um quarto componente foi incluído no modelo: o buffer episódico, onde as informações são armazenadas temporariamente para serem posteriormente reintegradas (BADDELEY, 2000).

Quase 40 anos, e muitas pesquisas após Baddeley e Hitch terem proposto seu modelo pela primeira vez, ele ainda é o modelo mais proeminente e consistente na literatura. No entanto, embora a definição de memória de trabalho possa ser um consenso - que ela se refere ao sistema responsável pelo armazenamento temporário e processamento da informação necessária para o desempenho de tarefas cognitivamente complexas (BADDELEY; HITCH, 1974; CANTOR; ENGLE, 1993; DANEMAN; CARPENTER, 1980, 1983; MASSON; MILLER, 1983; entre outros) - as perspectivas sob as quais ela é investigada diferem, bem como as evidências fornecidas por esses estudos (BARETTA, 2008).

Baddeley (1992) afirma que a pesquisa em MT pode ser dividida em dois tipos: o primeiro é baseado na abordagem psicométrica correlacional, enquanto o segundo é baseado na metodologia de dupla-tarefa e casos neuropsicológicos. A criação de tarefas envolvendo o processamento e armazenamento de informações na memória de trabalho cabe à abordagem psicométrica (TOMITCH, 2003). Um exemplo desse tipo de tarefa é o Reading Span Test (RST) ou Teste de Capacidade de Memória de Trabalho (TCMT), criado por Daneman e Carpenter (1980), a ser explicado posteriormente. A outra abordagem tem como objetivo analisar a estrutura do sistema de memória de trabalho, e para isso utiliza-se da metodologia de dupla-tarefa e de evidências de casos neuropsicológicos (TOMITCH, 2003). A pesquisa de Baddeley está incluída neste tipo de estudo, que consiste na realização de tarefas que envolvem processamento e armazenamento simultâneo de informações.

No que diz respeito às diferenças individuais na CMT, a abordagem psicométrica correlacional afirma que a capacidade de memória de trabalho diverge entre os indivíduos e que essas diferenças possibilitam prever seu desempenho em tarefas cognitivas (DANEMAN; CARPENTER, 1980; JUST; CARPENTER, 1992). De acordo com essa abordagem, indivíduos com CMT maior têm melhor desempenho em tarefas cognitivas do que indivíduos com CMT menor. A razão para tais diferenças é que indivíduos com maior CMT são capazes de reter e processar maiores quantidades de informações que são significativas para a realização de tarefas complexas, melhorando seu desempenho nas mesmas (WHITNEY; RITCHIE; CLARK, 1991; MCNAMARA; SCOTT, 2001). 


\section{Memória de Trabalho, Geração de Inferências e Compreensão Leitora em L2: Estudos Correlatos}

Pesquisas sobre diferenças individuais em CMT encontraram correlações positivas com uma ampla gama de tarefas cognitivas de ordem superior relacionadas à L1 (WHITNEY; RITCHIE; CLARK, 1991; SINGER et al., 1992; LINDERHOLM; BROEK, 2002; TOMITCH, 2003, entre outros). Por outro lado, muito menos estudos conduzidos em L2 e que apresentam correlações de CMT são encontrados na literatura da área. Esses estudos incluem correlação com a compreensão leitora (JOH; PLAKANS, 2017; WANG; LIN, 2019); construção da ideia principal em L1 e L2 (TORRES, 2003); compreensão leitora inferencial (ALPTEKIN; ERÇETIN, 2010); desempenho de escrita (BERGSLEITHNER, 2010); produção da fala (FINARDI; WEISSHEIMER, 2008); gramática e leitura (SAGARRA, 2017) e desenvolvimento da fala (WEISSHEIMER; MOTA, 2009).

Como a geração de inferências depende da integração de diferentes fontes de informação (do próprio texto e do conhecimento prévio dos leitores), como já mencionado, os requisitos de processamento e armazenamento de MT de compreensão da linguagem são essenciais para a geração de inferências (BARETTA, 2008). A capacidade de memória de trabalho permite aos leitores manter temas globais, integrar informações do texto e identificar os pontos principais do texto (DANEMAN; CARPENTER, 1980; CANTOR; ENGLE, 1993; LINDERHOLM, 2002), razão pela qual as diferenças individuais na CMT desempenham um papel importante na obtenção da compreensão leitora (JUST; CARPENTER, 1992).

Um estudo seminal conduzido por Trabasso e Magliano (1996) investigou os tipos de informações conscientemente disponíveis durante uma tarefa de compreensão leitora, e como tais informações são usadas inferencialmente para construir significado a partir do texto. Um modelo de compreensão consciente foi proposto pelos pesquisadores, por meio de um protocolo verbal do tipo think-aloud durante a compreensão de textos narrativos, pressupondo que a representação mental coerente do texto é construída através da união entre geração de inferências e memória. As verbalizações dos participantes a partir do protocolo verbal foram transcritas, analisadas e categorizadas como Paráfrases, Explicações (inferências concomitantes), Associações (inferências prévias), Predições (inferências futuras) ou Metacomentários. As frases também foram categorizadas de acordo com as operações de MT envolvidas: “(1) ativação de conhecimento relevante na memória de trabalho, (2) manutenção de informações na memória de trabalho e (3) recuperação de pensamentos anteriores a respeito do texto de um armazenamento de memórias de longo prazo" (TRABASSO; MAGLIANO, 1996, p.255, nossa tradução) ${ }^{5}$. Os resultados indicaram que as Explicações são a base da compreensão. Além disso, a incidência menos frequente de Previsões deu suporte à afirmação de que "a compreensão

No original: "(1) activation of relevant knowledge in working memory, (2) maintenance of information in working memory, and (3) retrieval of text prior thoughts from a long-term memory store" (TRABASSO; MAGLIANO, 1996, p.255). 
também é movida pela expectativa" (SCHANK; ABELSON, 1977 apud TRABASSO; MAGLIANO, 1996, p.273, tradução nossa) ${ }^{6}$.

Outro estudo relevante foi realizado por Zwaan e Brown (1996), os quais investigaram a proficiência (em L1 e L2) e a habilidade de compreensão (em L1), dois fatores que podem influenciar a construção do modelo situacional. Doze estudantes universitários falantes nativos de inglês e falantes não fluentes de francês como L2 participaram do estudo. Os participantes foram instruídos a pensar em voz alta enquanto liam narrativas em suas L1 e L2, executando, logo após, uma tarefa de agrupamento de verbos. Zwaan e Brown concluíram que quatro tipos de pensamentos ocorrem durante o protocolo verbal do tipo think aloud, e categorizaram os protocolos verbais dos participantes de acordo com essas taxonomias, que são Paráfrases, Explicações, Associações e Previsões. Eles previram que o número total de inferências geradas seria maior para L1 quando comparado com L2, devido ao fato de que o processamento de nível inferior consumiria mais recursos para a compreensão de L2. Além disso, eles levantaram a hipótese de que mais Paráfrases seriam observadas na compreensão de L2 do que em L1. Suas descobertas mostraram que um modelo situacional mais forte foi construído para os textos em L1 do que para os textos em L2. Dessa forma, foram feitas mais explicações para o texto L1 do que para o texto L2. Além disso, uma maior incidência de Explicações foi observada por participantes mais proficientes, que também construíram modelos situacionais mais fortes do que seus colegas menos proficientes.

O terceiro estudo seminal em que a presente pesquisa foi inspirada foi elaborado por Narvaez, Broek e Ruiz (1999). Eles conduziram uma pesquisa sobre a influência do propósito de leitura (estudo e entretenimento) na geração de inferências e na compreensão leitora. Vinte alunos de graduação, todos falantes nativos de inglês, atribuídos aleatoriamente a uma das duas condições (narrativa ou expositiva) participaram do estudo. Os participantes foram orientados a ler quatro textos, dois em voz alta (um texto narrativo e um texto expositivo) e dois para medidas de compreensão (um texto narrativo e um texto expositivo). As inferências geradas durante o protocolo de pausa dos dois primeiros textos foram avaliadas e categorizadas de acordo com um modelo baseado nos estudos de Zwaan e Brown (1996) e Trabasso e Magliano (1996). Os participantes também responderam perguntas de compreensão sobre os outros dois textos. Os resultados não mostraram correlação entre propósito de leitura e compreensão. O pensamento em voz alta, por outro lado, foi influenciado pelo propósito da leitura. Além disso, na leitura com objetivo de estudo observou-se um aumento no número de Repetições, Quebras de Coerência Baseadas no Conhecimento e Avaliações, que foram menos presentes na condição cujo propósito era de entretenimento. Segundo Zwaan e Brown (1996), esse padrão foi menos observado no texto narrativo em comparação com o texto expositivo, o que provavelmente indica que não apenas o propósito da leitura, mas também o tipo de texto influenciou o processo de geração de inferência dos leitores. No original: "understanding is also expectation driven” (SCHANK; ABELSON, 1977 apud TRABASSO;
MAGLIANO, 1996, p.273). 


\section{Método}

Participantes: Um grupo de 36 alunos do curso Técnico em Agropecuária Integrado ao Ensino Médio do Instituto Federal do Rio Grande do Sul (IFRS), Campus Sertão, concordou em participar da pesquisa. Os participantes eram falantes pré-intermediários de Inglês como Língua Estrangeira, conforme medido por um teste de proficiência em leitura? ${ }^{7}$.

Os 36 participantes do estudo principal foram aleatoriamente designados para um dos seguintes grupos: Grupo I: Pré-leitura Expositivo (ExpositivoPR) e Grupo II: Pré-leitura Narrativo (NarrativoPR). O grupo I foi exposto às atividades de pré-leitura antes da leitura do texto expositivo, mas não do narrativo; O Grupo II foi exposto às atividades de pré-leitura antes da leitura do texto narrativo, mas leu o texto expositivo sem qualquer atividade prévia ${ }^{8}$.

Instrumentos: Os textos. Para a coleta de dados foram utilizados um texto narrativo e um expositivo, com média de 250 palavras cada, os quais foram retirados do livro Inglês: Série Brasil da EFL High School (MARQUES, 2005).

O protocolo de pausa: O Protocolo de Pausa (CAVALCANTI, 1989) na versão adaptada por Tomitch (2003) foi utilizado neste estudo por parecer ter menos interferência no processo de leitura. Neste método de 'pensar em voz alta', os leitores são instruídos a ler o texto e parar sempre que encontrarem um problema ou algo que chame sua atenção, relatando-o. Além disso, um sinal vermelho é colocado no final de cada parágrafo, a fim de lembrar aos participantes que, naquele momento, eles devem parar e verbalizar sobre o que acabaram de ler. Os participantes também são instruídos a verbalizar o que lembrarem do texto como um todo, após terminarem de lê-lo.

O Teste de Capacidade de Memória de Trabalho: o Teste de Capacidade de Memória de Trabalho empregado neste estudo foi uma versão em português do teste Reading Span desenvolvido por Daneman e Carpenter (1980). Linck et al. (2013) recomendam o uso do Teste de Capacidade de Memória de Trabalho na L1 dos participantes, de forma a evitar variáveis intervenientes relacionadas à falta de proficiência na análise dos dados. A versão aqui utilizada foi criada por Tomitch (2003) e parcialmente adaptada por Bailer (2011), para que as sentenças do teste fossem mais adequadas à população adolescente, como também é o caso deste estudo.

O teste consistiu em 60 frases não relacionadas, com 13 a 17 palavras, terminando em uma palavra diferente, além de 9 frases adicionais para prática. As frases foram apresentadas uma a uma em forma de slides, e foram dispostas em conjuntos de três, em ordem crescente, ou seja, três conjuntos de duas sentenças, três conjuntos de três sentenças, três conjuntos de quatro, cinco e seis sentenças. A frase seguinte era mostrada logo após o participante ler a última palavra da frase na tela, seguindo a condição administrada pelo experimentador proposta por Daneman e Carpenter (1980), em que

Este estudo foi aprovado pelo Comitê de Ética em Pesquisa com Seres Humanos sob o número 1.599.459.

Mais informações em Roscioli (2017). 
o tempo necessário para ler as frases em voz alta era o único tempo permitido aos participantes.

Uma tela branca com pontos de interrogação indicava o fim do conjunto. Os participantes foram instruídos a ler as frases em voz alta, tentando compreendê-las, e a memorizar a última palavra de cada frase. Quando a tela com as aspas era mostrada, os participantes tinham que tentar lembrar as palavras finais de todas as frases do conjunto, na ordem em que apareceram. O número de pontos de interrogação apresentados na tela era correspondente ao número de palavras que deveriam ser lembradas. As sessões de treinamento e teste foram gravadas para posterior transcrição e pontuação.

Após a leitura de cada texto, os participantes foram orientados a responder um questionário escrito contendo questões de compreensão relacionadas ao respectivo texto. O questionário compreendeu tanto questões objetivas quanto subjetivas (a serem respondidas em português) sobre o texto lido.

As Perguntas de Compreensão Leitora. As questões foram criadas com base na subdivisão de Gagné, Yekovich e Yekovich (1993) dos Processos Componentes, que inclui decodificação, compreensão literal, compreensão inferencial e monitoramento da compreensão. A elaboração das questões também foi embasada na taxonomia de Pearson e Johnson (1978), que divide as questões em três categorias: textualmente explícitas (questões literais, cujas respostas podem ser encontradas na página), textualmente implícitas (embora as respostas ainda possam ser encontradas na página, para responder à pergunta, o leitor precisa usar seu conhecimento prévio, gerando inferências), e dependentes de conhecimento de mundo (a resposta é derivada do conhecimento prévio do leitor, não do texto). Este estudo incluiu as três categorias de questões mencionadas anteriormente na tarefa de compreensão de leitura.

\section{Pontuação e Procedimentos Estatísticos}

O Teste de Capacidade de Memória de Trabalho foi pontuado de duas formas: estrita e leniente. Para a pontuação estrita do teste, o intervalo de leitura foi determinado pelo nível no qual o participante era capaz de lembrar pelo menos duas sequências de um determinado conjunto de sentenças não relacionadas (DANEMAN; CARPENTER, 1980). Meio ponto foi dado quando o participante passou da primeira sequência em um determinado nível (MASSON; MILLER, 1983; TOMITCH, 2003). Por exemplo, se um participante relembrasse corretamente todas as palavras na ordem certa nos três conjuntos de 2 frases, e nos três conjuntos de 3 frases lembrasse corretamente de apenas um grupo, este participante receberia meio ponto, e sua capacidade de leitura seria de 2,5. O teste terminava quando o participante reprovasse nas três séries em dois níveis subsequentes. ${ }^{9}$

Todos os participantes realizaram o TCMT como um todo, ou seja, até os três conjuntos de seis sentenças; entretanto, para o método de pontuação estrita, as respostas foram consideradas apenas até o ponto em que fossem capazes de lembrar de pelo menos um conjunto em um determinado nível. 
As respostas dos participantes no Teste de Capacidade de Memória de Trabalho também foram pontuadas de forma leniente. Este método considerou o número total de palavras lembradas em todos os conjuntos, independente da ordem. Na pontuação leniente, o número total de palavras lembradas corretamente foi considerado e "o terço superior e inferior da distribuição de frequência do total de palavras lembradas por cada participante foi usado para distinguir leitores de baixa e alta CMT." (LINDERHOLM; BROEK, 2002, p.779). ${ }^{10}$

O procedimento de categorização de inferência adotado neste estudo foi baseado no estudo realizado por Narvaez, Broek e Ruiz (1999), cuja pesquisa foi baseada em dois estudos anteriores propostos por Zwaan e Brown (1996) e Trabasso e Magliano (1996). A fim de categorizar as inferências dos leitores, os enunciados dos participantes foram divididos em blocos de ideias (GERBER; TOMITCH, 2008; CALDART, 2012). Em seguida, as sentenças dos participantes foram categorizadas de acordo com o modelo de categorização de inferências de Narvaez, Broek e Ruiz (1999), como Repetições, Explicações, Associações, Avaliações, Previsões, Quebras de Coerência Baseadas no Conhecimento ou Quebras de Coerência Baseadas no Texto. Para o propósito deste estudo, foram utilizadas duas outras categorias, baseadas em Zwaan e Brown (1996): Traduções Incorretas e Tentativas de Tradução.

As questões de compreensão leitora foram corrigidas e pontuadas por três avaliadores independentes e os resultados de ambos os grupos foram comparados. Os avaliadores foram orientados a corrigir as respostas da seguinte forma: 1 ponto se a resposta estivesse totalmente correta; 0,5 pontos se a resposta estivesse parcialmente correta; 0 pontos se a resposta estivesse incorreta. Eles receberam instrução de julgar apenas o conteúdo das respostas, ou seja, erros gramaticais deveriam ser desconsiderados.

Os testes de coeficiente alfa de Cronbach foram executados a fim de verificar a consistência interna das pontuações nas respostas às questões de compreensão dos textos expositivo e narrativo.

\section{Resultados e Discussão}

Teste de Capacidade de Memória de Trabalho. Seguindo o método de pontuação estrito, 11 participantes foram categorizados com capacidade de memória de trabalho entre 3 e 3,5 (spans intermediários, de acordo com Daneman e Carpenter (1980)), enquanto 25 foram considerados leitores de baixa CMT, com span abaixo de 2,5. Nenhum participante atingiu um span de 4 ou 5 e, portanto, este método de pontuação não teve nenhum indivíduo de alta CMT.

Quanto ao método de pontuação leniente, onze participantes lembraram de 31 a 38 palavras e foram categorizados como spans altos (LINDERHOLM; BROEK, 2002). No original: "the upper and lower third of the frequency distribution of total words recalled by each participant was
used to distinguish low- from high-WMC readers." (LINDERHOLM; BROEK, 2002, p.779). 
Quinze participantes foram considerados spans intermediários, com variação entre 26 e 30 palavras. Dez participantes foram classificados como leitores de baixa CMT, que lembraram de 19 a 25 palavras.

Geração de Inferências. Um total de 4.775 inferências foi gerado pelos participantes durante o protocolo de pausa, sendo 2.342 para o texto narrativo e 2.433 para o expositivo. Em relação ao número total de inferências geradas para cada texto, e levando em consideração o tipo de texto, os resultados deste estudo não corroboram os de Graesser, (1981), Britton et al. (1983), Graesser e Kreuz (1993), Trabasso e Magliano (1996), Narvaez, Broek e Ruiz (1999), Narvaez (2002), entre outros, que fornecem evidências de que os leitores geram consideravelmente mais inferências ao ler narrativas. É possível observar que neste estudo os participantes geraram mais inferências para o texto expositivo. Mais especificamente, gerou-se um total de 2.342 inferências para o texto narrativo, em comparação com 2.433 para o expositivo. Além disso, nos estudos realizados por Horiba (2000), Baretta (2008) e Caldart (2012), e de acordo com os resultados do presente estudo, as inferências foram geradas com maior frequência para o texto expositivo, quando comparado ao narrativo. Segundo Horiba (2000) e Baretta (2008), uma possível interpretação para os resultados discrepantes reside no texto utilizado em tais experimentos, que não foram tão exigentes quanto os de estudos anteriores (TRABASSO; MAGLIANO, 1996; NARVAEZ; BROEK; RUIZ, 1999, entre outros). Em outras palavras, o fluxo de leitura dos textos expositivos menos exigentes foi provavelmente muito semelhante ao fluxo de leitura das narrativas, devido às características específicas dos textos, como familiaridade com o tema e ausência de introdução de novos conceitos.

A relação entre geração de inferências e compreensão leitora: análise qualitativa. Considerando a relação entre tipo de inferência e desempenho nas questões de compreensão leitora, observou-se que para vinte e seis dos trinta e seis participantes, ou $72 \%$, o número de inferências foi inferior para o texto em que tiveram melhor desempenho, ou seja, menos inferências foram necessárias para construir coerência. Em outras palavras, os participantes geraram mais inferências para tentar entender o texto. Em relação a isso, Ericsson e Simon (1993) argumentam que para que os leitores forneçam dados durante os protocolos de pausa é necessário fazer uso de tarefas que exijam o controle estratégico e monitorado dos leitores, de modo que alguns processos automáticos sejam 'desautomatizados'. Nesse sentido, textos considerados fáceis pelos leitores são lidos automaticamente e, consequentemente, ficam menos disponíveis para relato consciente. Segundo Pressley e Afflerbach (1995), textos mais desafiadores fornecem mais dados porque exigem uma leitura mais controlada e cuidadosa, como foi o caso deste estudo, em que os participantes geraram mais inferências para o texto que lhes era mais desafiador.

Levando-se em consideração os participantes com as pontuações médias mais altas e aqueles com as pontuações médias mais baixas nas questões de compreensão leitora, é possível observar que a quantidade de inferências não variou tanto entre eles, então o que pode ter afetado seu desempenho foram os tipos das inferências geradas. 
Na tentativa de encontrar um padrão de geração de inferência entre participantes com maior e menor capacidade de compreensão, os 36 participantes foram separados em dois grupos, de acordo com sua pontuação nas questões de compreensão leitora, com 18 indivíduos em cada grupo (maior e menor pontuação). As maiores pontuações variaram de 9,63 a 7.965, enquanto as menores variaram de 4,9 a 7,96. Após essa separação, os resultados foram mais evidentes: o número total de inferências foi maior para os leitores com menor capacidade de compreensão (2517) quando comparados com os de maior capacidade de compreensão (2258). No entanto, os resultados mais interessantes relacionam-se aos tipos de inferências que predominaram em cada grupo. Enquanto os leitores proficientes tiveram a maior incidência de Repetições e Explicações, os leitores menos proficientes geraram mais Associações, Previsões, Avaliações, Quebras de Coerência Baseadas no Texto, Quebras de Coerência Baseadas no Conhecimento, Tentativas de Tradução e Traduções Incorretas. A comparação dos tipos de inferências geradas por leitores com maior capacidade de compreensão e leitores com menor capacidade pode ser melhor visualizada na imagem 1 a seguir.

Imagem 1 - Tipos de inferências gerados por leitores mais e menos proficientes

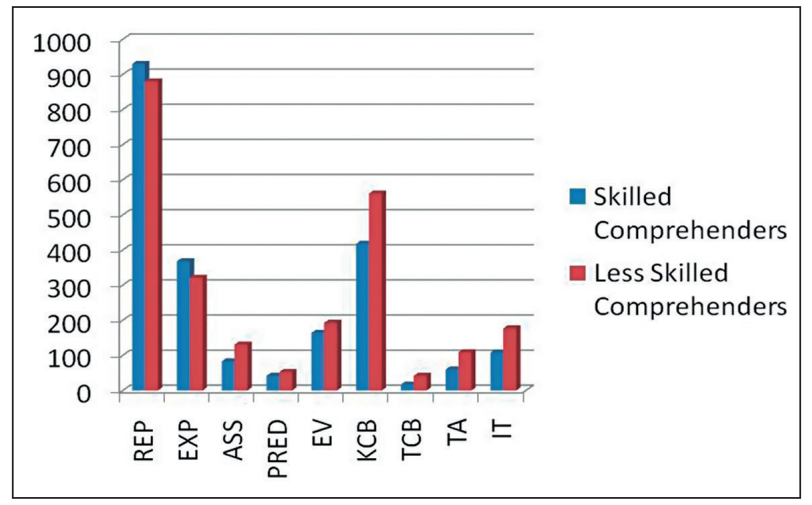

Fonte: Dados da pesquisa.

Como afirmado anteriormente, acredita-se que as Explicações estejam mais relacionadas à compreensão (GRAESSER; SINGER; TRABASSO, 1994; TRABASSO; MAGLIANO, 1996; ZWAAN; BROWN, 1996; NARVAEZ; BROEK; RUIZ, 1999; MAGLIANO; TRABASSO; GRAESSER, 1999, entre outros), por serem o principal meio através do qual a coerência é alcançada (BROEK; RISDEN; HUSEBYEHARTMAN, 1995 apud TRABASSO; MAGLIANO, 1996). Na verdade, a habilidade de gerar inferências Explicativas é o que diferencia os leitores mais proficientes (aqueles que apresentam nível de compreensão superior) dos menos proficientes (ZWAAN; BROWN, 1996).

Além disso, Trabasso e Magliano (1996) afirmam que a Paráfrase (que neste estudo foi incluída no tipo de inferência denominado Repetições) "aumentou a disponibilidade 
de uma frase para uso futuro durante a compreensão"11 (TRABASSO; MAGLIANO, 1996, p.282), o que permite a construção de uma "base de texto mais coerente e completa"12 ( TRABASSO; MAGLIANO, 1996, p.320). Além disso, as Repetições estão relacionadas ao objetivo de estudo, o que "corrobora as avaliações dos leitores sobre seus próprios processos de leitura, em particular sua percepção de que a leitura escolar (estudo) envolve mais releituras e tentativas de integração"13 (LORCH; LORCH; KLUSEWITZ, 1993, p. 493). Portanto, como esses dois tipos de inferências (Explicações e Repetições) foram os únicos gerados em maior quantidade pelos leitores mais proficientes, tal resultado parece corroborar os de Trabasso e Magliano (1996), Zwaan e Brown (1996) e Narvaez, Broek e Ruiz (1999), que observaram Explicações e Repetições como estando relacionadas à compreensão de leitura bem-sucedida.

A maior incidência dos outros tipos de inferências (ou seja, Associações, Previsões, Avaliações, Quebras de Coerência Baseadas no Texto, Quebras de Coerência Baseadas no Conhecimento, Tentativas de Tradução e Traduções Incorretas) foi observada nos 18 participantes que pertenciam ao grupo de leitores mais proficientes. Esses resultados também são corroborados pela literatura (TRABASSO; MAGLIANO, 1996; ZWAAN; BROWN, 1996; NARVAEZ; BROEK; RUIZ, 1999), como será explicado a seguir. Segundo Zwaan e Brown (1996), como as Associações não estão diretamente relacionadas ao texto, elas tendem a ser incorretas ou irrelevantes, razão pela qual leitores proficientes são mais seletivos na geração desse tipo de inferência. Quanto às Previsões, elas não são consideradas eficazes para a compreensão porque podem estar erradas. No que diz respeito aos comentários Avaliativos, eles não estão diretamente relacionados à construção do modelo situacional, uma vez que tal modelo é a representação do que o texto trata, enquanto as Avaliações abrangem as percepções dos leitores sobre o conteúdo do texto, sua escrita, ou o estado de espírito deles mesmo durante a leitura (NARVAEZ; BROEK; RUIZ, 1999). E, finalmente, Quebras de Coerência Baseadas no Texto, Quebras de Coerência Baseadas no Conhecimento, Tentativas de Tradução e Traduções Incorretas estão diretamente relacionadas com a dificuldade de compreensão. Portanto, parece plausível supor que uma maior incidência desses tipos de inferências esteja relacionada a uma compreensão menos bem-sucedida (ZWAAN; BROWN, 1996; NARVAEZ; BROEK; RUIZ, 1999).

Correlações entre memória de trabalho e geração de inferências. Os índices de correlação de Spearman mostram resultados semelhantes para ambos os grupos, sendo que apenas uma relação estatisticamente significativa foi encontrada entre a capacidade de memória de trabalho (CMT) de cada um dos grupos e seu processo de geração de inferências. Em relação ao grupo ExpositivoPR, foi obtida correlação positiva,

\footnotetext{
11 No original: "increased the availability of a sentence for future use during comprehension" (TRABASSO; MAGLIANO, 1996, p.282)

12 No original "more coherent and complete textbase" (TRABASSO; MAGLIANO, 1996, p.320).

13 No original: “corroborates readers' assessments of their own reading processes, in particular their perception that school (study) reading involves more rereading and attempts at integration." (LORCH; LORCH; KLUSEWITZ, 1993, p.493).
} 
moderada, estatisticamente significativa $(\mathrm{p}<0,05)$ entre as Explicações e a CMT em relação ao texto expositivo $(\mathrm{r}=0,506, \mathrm{p}=0,038)$. Já para o grupo NarrativoPR, foi encontrada correlação positiva, moderada, estatisticamente significativa $(\mathrm{p}<0,05)$ entre Repetições e CMT em relação ao texto expositivo $(\mathrm{r}=0,468, \mathrm{p}=0,043)$. No que se refere a todas as outras variáveis, os testes de correlação estatística executados não apresentaram relações significativas em nenhum dos grupos experimentais.

O coeficiente de correlação de Pearson e os índices de correlação de Spearman mostram resultados semelhantes para ambos os grupos, sendo que apenas uma relação estatisticamente significativa foi encontrada entre a CMT e a geração de inferências de cada um dos grupos. Em relação ao grupo ExpositivoPR, foi obtida correlação positiva, próxima a moderada, estatisticamente significativa $(\mathrm{p}<.05)$ entre as Explicações e a CMT em relação ao texto expositivo $(\mathrm{r}=.497, \mathrm{p}=.043)$. Já para o grupo NarrativoPR, foi encontrada correlação positiva, moderada, estatisticamente significativa $(p<0,05)$ entre Repetições e CMT em relação ao texto expositivo $(\mathrm{r}=0,587, \mathrm{p}=0,008)$. Não foram encontradas relações significativas no que concerne às outras variáveis em nenhum dos grupos experimentais considerando-se o método de pontuação leniente do Teste de Capacidade de Memória de Trabalho.

Os índices de correlação de Spearman mostram resultados semelhantes para ambos os grupos sendo que apenas uma relação estatisticamente significativa foi encontrada entre a CMT e a geração de inferências de cada um dos grupos, no que se refere ao texto narrativo. Semelhante aos resultados apresentados anteriormente, em relação ao texto expositivo, para o grupo ExpositivoPR, foi obtida uma correlação positiva, moderada, estatisticamente significativa $(\mathrm{p}<0,05)$ entre Explicações e CMT em relação ao texto narrativo $(\mathrm{r}=0,522, \mathrm{p}=.032)$. Já para o grupo NarrativoPR, foi encontrada uma correlação positiva, próxima a moderada, estatisticamente significativa $(\mathrm{p}<0,05)$ entre Repetições e CMT em relação ao texto narrativo $(\mathrm{r}=0,484, \mathrm{p}=0,036)$. No que diz respeito a todas as outras variáveis, os testes de correlação estatística executados não mostraram relação significativa em nenhum dos grupos experimentais.

O coeficiente de correlação de Pearson e os índices de correlação de Spearman não mostram interação estatisticamente significativa entre qualquer uma das variáveis de geração de inferência e CMT em relação ao texto narrativo por ambos os grupos experimentais $(\mathrm{p}>$.05) com relação ao método de pontuação leniente.

Já com relação aos resultados dos testes estatísticos correlacionais entre geração de inferência e CMT, considerando-se os dois métodos de pontuação do TCMT empregados neste estudo (estrito e leniente), duas correlações foram observadas para o texto expositivo, no que concerne a relação entre geração de inferências e CMT, sendo um para cada um dos grupos. Essa correlação foi igual para ambos os métodos de pontuação, o que se explica pelo fato de os dois métodos (leniente e estrito) se correlacionarem positivamente neste estudo, e significando que medem os mesmos construtos. Com relação ao Grupo ExpositivoPR (GI), foi encontrada correlação positiva, moderada e estatisticamente significativa entre Explicações e CMT. Tal resultado não é surpreendente, uma vez que é de conhecimento entre os estudiosos da 
área da leitura que leitores mais e menos proficientes podem ser diferenciados por sua capacidade de gerar inferências explicativas (ZWAAN; BROWN, 1996; TRABASSO; MAGLIANO, 1996; BROEK; LORCH, 1993; GRAESSER; SINGER; TRABASSO, 1994, entre outros). Isso pode ser explicado pela afirmação de que Explicações ajudam a "integrar a informação da frase em uma representação de memória mais coerente" 14 (MAGLIANO; GRAESSER; TRABASSO, 1999, p.616, tradução nossa), resultando também em uma melhor retenção do texto. Além disso, foi observada uma melhor retenção do texto entre os participantes que geraram Explicações durante a leitura (TRABASSO; MAGLIANO, 1996; MAGLIANO; GRAESSER; TRABASSO, 1999).

Ainda com relação ao texto expositivo, foi encontrada correlação positiva, moderada e estatisticamente significativa entre Repetições e CMT para o Grupo NarrativoPR (GII). Esse resultado é provavelmente decorrente do tipo de texto, pois segundo Narvaez, Broek e Ruiz (1999) o comportamento do tipo estudo parece ser invocado por textos expositivos, o que inclui a geração de Repetições.

Quanto ao texto narrativo, as mesmas correlações foram encontradas entre Explicações (Grupo ExpositivoPR) e Repetições (Grupo NarrativoPR) e CMT, mas apenas levando-se em consideração o método de pontuação estrito. No entanto, é interessante observar que embora ambos os tipos de texto apresentassem correlações entre CMT e Repetições / Explicações, as correlações foram mais fortes para o texto narrativo, quando comparado com o expositivo. A correlação entre CMT (pontuação estrita) e geração de inferências para o texto expositivo foi $r=0,506$ (p.038), enquanto esta mesma correlação foi $r=522$ (p.032) para o texto narrativo. Da mesma forma, a correlação entre Repetições e CMT para o texto expositivo foi $r=468$ (p.043) em comparação com $r=484$ (p.036) para o texto narrativo. O tipo de texto pode ter tido influência nos resultados acima mencionados, pois textos narrativos instigam diferentes comportamentos de leitura quando comparados aos textos expositivos (NARVAEZ; BROEK; RUIZ, 1999; TRABASSO; MAGLIANO, 1996), principalmente por serem mais fáceis de entender e promoverem mais inferências em geral, especialmente Explicações.

Não foram observadas correlações estatisticamente significativas entre geração de inferências e CMT (pontuação leniente), para o texto narrativo. A falta de resultados significativos para a pontuação leniente pode ser devido ao tamanho da amostra, que é pequena e quanto menor a amostra, mais difícil observar resultados estatisticamente significativos. De acordo com Juffs e Harrington (2011, p.145) “a ausência de uma correlação significativa, especialmente em comparação com estudos anteriores, pode refletir meramente o tamanho da amostra" 15 .

Correlações entre memória de trabalho e compreensão leitora. O coeficiente de correlação de Pearson e os índices de correlação de Spearman mostram resultados

14 No original: "integrate the sentence information into a more coherent memory representation" (MAGLIANO; GRAESSER; TRABASSO, 1999, p.616).

15 No original: "the absence of a significant correlation, especially in comparison to previous studies, may merely reflect direct sample sizes” (JUFFS; HARRINGTON, 2011, p.145). 
diferentes para cada um dos grupos experimentais. Embora não tenham sido encontradas correlações estatisticamente significativas para o grupo ExpositivoPR ( $\mathrm{n}=17)$, no que se refere à relação entre a compreensão leitora dos grupos, tanto do texto expositivo quanto do narrativo e a CMT dos participantes $(\mathrm{p}>.05)$, os resultados obtidos com os testes estatísticos para o grupo NarrativoPR $(n=19)$ mostraram correlações moderadas, positivas e estatisticamente significativas $(\mathrm{p}<.05)$, entre a compreensão leitora do grupo tanto para o texto narrativo quanto para o expositivo e sua capacidade de memória de trabalho no que diz respeito ao método de pontuação estrito $(r=0,463, p=0,046)$, mas não ao método leniente ( $\mathrm{p}>0,05)$.

O coeficiente de correlação de Pearson e os índices de correlação de Spearman mostram resultados diferentes para cada um dos grupos experimentais. Enquanto nenhuma correlação estatisticamente significativa foi encontrada para o grupo ExpositivoPR $(n=14)$, no que se refere à relação entre a compreensão leitora dos grupos, tanto do texto narrativo quanto do expositivo e a CMT dos participantes ( $\mathrm{p}>$.05), os resultados obtidos com os testes estatísticos do grupo NarrativoPR (n =18) mostraram correlações moderadas, positivas e estatisticamente significativas $(\mathrm{p}<.05)$ entre a compreensão leitora do grupo, tanto para o texto narrativo quanto para o texto expositivo e sua CMT, considerando-se o método de pontuação estrito $(\mathrm{r}=0,615, \mathrm{p}=0,007)$, mas não o método leniente $(\mathrm{p}>0,05)$, quando os outliers não foram fatorados.

Os resultados dos testes estatísticos foram diferentes para os dois grupos (ExpositivoPR e NarrativoPR) quanto à correlação entre CMT e compreensão leitora. Não foi encontrada nenhuma correlação estatística entre essas duas variáveis para o Grupo I (ExpositivoPR). No entanto, para o Grupo II (NarrativoPR), foi observada uma correlação moderada, positiva e estatisticamente significativa entre a compreensão leitora dos participantes e sua CMT. No entanto, tal correlação foi encontrada apenas quando considerado o método estrito de pontuação, não o método leniente.

Uma possível explicação para a falta de resultados estatisticamente significativos do Grupo ExpositivoPR é que ele possui menor quantidade de participantes $(n=17)$, em comparação com o Grupo NarrativoPR $(n=19)$, o que é uma pequena diferença, mas quando se trata de análise estatística, conforme apontado anteriormente, quanto menor a amostra, mais difícil é o alcance de significância estatística.

$\mathrm{O}$ fato de apenas o método de pontuação estrito apresentar resultados estatisticamente significativos, e não o leniente, pode ser explicado pelo fato de que "o escore leniente, por sua natureza, está menos relacionado ao controle do que o escore estrito, que reflete processos controlados"16 (FINARDI; WEISSHEIMER, 2008, p.380) Como as limitações da CMT provavelmente estão mais relacionadas ao controle e menos relacionadas aos processos automáticos, a correlação entre memória de trabalho e compreensão leitora só foi observada para o método estrito de pontuação.

\footnotetext{
16 No original: "the Lenient score, for its nature, is less related to control than the Strict score which reflects controlled processes.” (FINARDI; WEISSHEIMER, 2008, p.380).
} 
No que se refere ao resultado observado para o Grupo II (NarrativoPR), que apresentou correlação moderada, positiva e estatisticamente significativa entre CMT e compreensão leitora, é possível supor que mais uma vez a atividade audiovisual de pré-leitura pode ter influenciado, pois a correlação foi encontrada tanto para o texto narrativo quanto para o expositivo. Portanto, os esquemas deste grupo provavelmente foram ativados para ambos os textos, o que enriqueceu sua compreensão (TOMITCH, 1991).

Levando em consideração que textos expositivos são considerados mais difíceis de entender do que os narrativos (NARVAEZ; BROEK; RUIZ, 1999; TRABASSO; MAGLIANO, 1996; KRAAL, A. et al., 2018), após analisar os dados de participantes com menor e maior CMT, os resultados de suas perguntas de compreensão leitora e a quantidade de inferências geradas para cada texto, é possível observar que os resultados do presente estudo não corroboram as evidências encontradas na literatura, que sustentam que as diferenças entre participantes com maior ou menor CMT são mais evidentes quando a tarefa é difícil, ao contrário de tarefas consideradas fáceis, nas quais ambos os grupos apresentarão resultados semelhantes (JUST; CARPENTER, 1992; BUDD; WHITNEY; TURLEY, 1995; BARETTA, 2008, entre outros). Alguns dos participantes com maiores pontuações no TCMT tiveram melhor desempenho nas questões de compreensão leitora do texto narrativo, enquanto outros tiveram melhor desempenho nas questões relacionadas ao texto expositivo. $\mathrm{O}$ mesmo aconteceu com os participantes com as menores pontuações no TCMT. Da mesma forma, o número de inferências gerado participantes com maior e menor CMT não seguiu um padrão. Uma possível explicação para tais resultados pode estar relacionada às atividades de pré-leitura que ativaram os esquemas dos participantes, tornando os dois textos semelhantes no nível de dificuldade. Portanto, o que pode ter desempenhado um papel nos resultados dos participantes nas questões de compreensão leitora e nas inferências geradas foi provavelmente o interesse individual pelos tópicos dos textos.

Correlações entre compreensão leitora e geração de inferências. O coeficiente de correlação de Pearson e os índices de correlação de Spearman mostram resultados semelhantes para cada um dos grupos experimentais. Uma correlação negativa, moderada e estatisticamente significativa $(\mathrm{p}<.05)$ foi encontrada entre Tentativa de Tradução e compreensão leitora para o grupo Narrativ0PR $(\mathrm{r}=-.642, \mathrm{p}=.003)$. Nenhuma correlação estatisticamente significativa foi encontrada para as demais variáveis (Repetições, Explicações, Associações, Previsões, Avaliações, Quebras de Coerência Baseadas no Conhecimento, Quebras de Coerência Baseadas no Texto, Traduções Incorretas e Total) para ambos os grupos no que se refere a relação entre a compreensão leitora dos grupos e os tipos de inferências gerados para o texto expositivo.

Em suma, apenas uma correlação negativa moderada, estatisticamente significativa, foi encontrada entre a geração de inferências e a compreensão leitora do texto expositivo. A correlação negativa encontrada foi entre Tentativa de Tradução e compreensão leitora para o grupo NarrativoPR (GII). Além disso, as Quebras de Coerência Baseadas no Texto quase alcançaram significância $(\mathrm{r}=-.415, \mathrm{p}=.077)$ e, portanto, também merecem 
atenção. Esses resultados mostram que quanto menos os participantes tentaram traduzir o texto e quanto menos quebras de coerência relacionadas ao texto ocorreram, maior foi a compreensão leitora

O coeficiente de correlação de Pearson e os índices de correlação de Spearman mostram que não foram encontradas correlações estatisticamente significativas para todas as variáveis do grupo ExpositivoPR no que se refere à relação entre a compreensão leitora do grupo e os tipos de inferências geradas para o texto narrativo $(p>.05)$. Quanto ao segundo grupo experimental - o grupo NarrativoPR - foram encontradas três relações estatisticamente significativas $(\mathrm{p}<0,05)$, que serão discutidas a seguir: uma relação negativa, próxima a forte, entre Avaliação e compreensão leitora do texto narrativo pelo grupo $(\mathrm{r}=$-. 690, $\mathrm{p}=.001)$; uma relação negativa e moderada entre Tentativa de Tradução e compreensão leitora do texto narrativo pelo grupo $(\mathrm{r}=-.548, \mathrm{p}=.015)$; e, por fim, foi encontrada uma relação negativa e moderada entre Tradução Incorreta e compreensão leitora do texto narrativo pelo grupo $(r=.-460, p=.048)$.

Em suma, não foram observadas correlações estatisticamente significativas entre geração de inferências e compreensão leitora para o texto narrativo quando considerado o Grupo ExpositivoPR (Grupo I). Já para o Grupo NarrativoPR (Grupo II), foram encontradas três correlações estatisticamente significativas entre geração de inferência e compreensão leitora para o texto narrativo: uma correlação negativa, próxima a forte, entre Avaliação e compreensão leitora; uma relação negativa e moderada entre Tentativa de Tradução e compreensão leitora; e uma correlação negativa moderada entre Tradução Incorreta e compreensão leitora do texto narrativo. Além disso, as Quebras de Coerência Baseadas no Texto e a compreensão leitora do texto narrativo quase alcançaram significância $(r=-.433, p=.064)$ e, portanto, sua correlação negativa também merece atenção.

Tais correlações negativas eram esperadas, pois Avaliações, Quebras de Coerência Baseadas no Texto, Tentativas de Tradução e Traduções Incorretas não são inferências integrativas. Conforme mencionado anteriormente, as Avaliações incluem a opinião do leitor sobre o assunto do texto ou seu estado de espírito durante a atividade de leitura. Quebras de Coerência Baseadas no Texto, Tentativas de Tradução e Traduções Incorretas, quando feitas em excesso, podem ter um impacto negativo no fluxo de leitura e, portanto, prejudicar a compreensão leitora, de forma que quanto menor a incidência desses três tipos de inferências, melhor para a compreensão de leitura.

\section{Considerações Finais}

Esta pesquisa teve como objetivo investigar se existe relação entre a Capacidade de Memória de Trabalho de estudantes brasileiros do Ensino Médio Técnico, geração de inferência e compreensão leitora em L2. De modo geral, os resultados deste estudo se mostram favoráveis à relação entre CMT, geração de inferência e compreensão leitora. Entre os principais achados da presente pesquisa estão as seguintes: 
Achado 1 - CMT (conforme medido pelo método de pontuação estrito) se correlaciona significativamente com a compreensão leitora. Os resultados obtidos com os testes estatísticos para o GII (NarrativoPR) mostraram correlações moderadas, positivas e estatisticamente significativas entre a compreensão leitora dos grupos tanto dos textos expositivos quanto dos narrativos - e sua capacidade de memória de trabalho, no que se refere ao método de pontuação estrito $(\mathrm{p}<.05)$, mas não com relação ao método leniente ( $\mathrm{p}>$.05). Em relação a esses resultados, é possível supor que a atividade de pré-leitura audiovisual realizada pelo Grupo II (NarrativoPR) possa ter influenciado, pois a correlação foi encontrada tanto para a narrativa quanto para o texto expositivo. Portanto, os esquemas deste grupo foram provavelmente ativados para ambos os textos, o que enriqueceu sua compreensão.

Achado 2 - a CMT correlaciona-se positivamente com Repetições e inferências Explicativas. Em relação ao Grupo ExpositivoPR (GI), foi encontrada correlação positiva, moderada e estatisticamente significativa entre as Explicações e a memória de trabalho para o texto expositivo. Esse resultado não é surpreendente, porque é de conhecimento entre os estudiosos da área que leitores mais ou menos proficientes podem ser diferenciados por sua capacidade de gerar inferências explicativas (ZWAAN; BROWN, 1996; TRABASSO; MAGLIANO, 1996; BROEK; LORCH, 1993, GRAESSER; SINGER; TRABASSO, 1994, entre outros). Ainda em relação ao texto expositivo, foi encontrada correlação positiva, moderada e estatisticamente significativa entre Repetições e memória de trabalho para o Grupo NarrativoPR (GII). Esse resultado está provavelmente relacionado ao tipo de texto, pois segundo Narvaez, Broek e Ruiz (1999, p.493) "os textos expositivos parecem evocar comportamentos do tipo estudo"17, que incluem a geração de Repetições.

Quanto ao texto narrativo, as mesmas correlações foram encontradas entre Repetições (Grupo ExpositivoPR) e Explicações (Grupo NarrativoPR) e memória de trabalho, mas apenas quando considerado o método estrito de pontuação. No entanto, é interessante observar que embora ambos os tipos de texto apresentassem correlações entre memória de trabalho e Repetições / Explicações, as correlações foram mais fortes para o texto narrativo, quando comparado com o expositivo. Dessa forma, é possível que o tipo de texto tenha influenciado os resultados previamente apresentados, pois, como mencionado anteriormente, os textos narrativos instigam diferentes comportamentos de leitura quando comparados aos textos expositivos (NARVAEZ; BROEK; RUIZ, 1999; TRABASSO; MAGLIANO, 1996), especialmente por estes serem mais fáceis de compreender.

Achado 3 - Leitores menos proficientes geram mais inferências em geral, mas leitores mais proficientes geram mais inferências Explicativas, que estão diretamente ligadas à compreensão. O número total de inferências foi maior para os leitores menos proficientes do que para os mais proficientes. No que diz respeito aos tipos de inferências geradas pelos participantes, os leitores mais proficientes tiveram a

17 No original: “expository texts seem to evoke study-type behaviors” (NARVAEZ; BROEK; RUIZ, 1999, p.493). 
maior incidência de Repetições e Explicações, enquanto os leitores menos proficientes geraram mais Associações, Previsões, Avaliações, Quebras de Coerência Baseadas no Texto, Quebras de Coerência Baseadas no Conhecimento, Tentativas de Tradução e Traduções Incorretas. Como afirmado anteriormente, acredita-se que as Explicações estejam mais relacionadas à compreensão (GRAESSER; SINGER; TRABASSO, 1994; TRABASSO; MAGLIANO, 1996; ZWAAN; BROWN, 1996; NARVAEZ; BROEK; RUIZ, 1999; MAGLIANO; GRAESSER; TRABASSO, 1999, entre outros), umas vez que estas são o principal meio para que a coerência seja alcançada (BROEK; RISDEN, 1995). Na verdade, a habilidade de usar inferências Explicativas é o que diferencia os leitores mais proficientes dos menos proficientes, de acordo com Zwaan e Brown (1996). Portanto, como esses dois tipos de inferências (Explicações e Repetições) foram os únicos em que os leitores mais proficientes tiveram o maior número, esse resultado parece corroborar os de Trabasso e Magliano (1996), Zwaan e Brown (1996) e Narvaez, Broek e Ruiz (1999), que observaram Explicações e Repetições como estando relacionadas à compreensão leitora bem-sucedida.

\section{Implicações Pedagógicas}

É fato que as diferenças individuais desempenham um grande papel na aprendizagem dos alunos. No caso do inglês como L2, fatores como proficiência no idioma, conhecimento prévio, habilidades de leitura, motivação e CMT devem ser levados em consideração por qualquer professor de língua estrangeira antes de planejar suas aulas. Embora a CMT não seja medida na escola, os professores precisam estar cientes de seu impacto na aprendizagem. É importante que os textos selecionados para as aulas de leitura sejam adequados ao nível de proficiência dos alunos, de forma que a CMT não fique sobrecarregada com processos de leitura de nível inferior. Além disso, levando-se em consideração que a geração de inferências ajuda os alunos a construir significado a partir dos textos, entender como os leitores com maior e menor CMT geram inferências e como a geração de inferências afeta a compreensão pode ajudar os professores a auxiliarem seus alunos a se tornarem leitores mais proficientes.

Acredita-se que os achados desta pesquisa contribuam, ainda que em pequena escala, para a compreensão do papel da capacidade da memória de trabalho na geração de inferências e na compreensão leitora de alunos brasileiros de Ensino Médio Técnico. Além disso, espera-se que este estudo some às pesquisas existentes sobre as diferenças individuais na CMT e no desempenho em leitura, não só na área educacional, mas também em áreas como compreensão do discurso, linguística textual e computacional e psicolinguística, fornecendo achados que contribuam para um melhor entendimento do processo de compreensão leitora. 


\section{Agradecimentos}

Gostaríamos de agradecer o Instituto Federal de Educação, Ciência e Tecnologia do Rio Grande do Sul por ter possibilitado a realização desta pesquisa por meio do afastamento para capacitação da primeira autora.

ROSCIOLI, D.; TOMITCH, L. The Influence of Working Memory Capacity on Inference Generation and Reading Comprehension. Alfa, São Paulo, v.66, 2022.

- ABSTRACT: This research aimed at investigating whether there is a relationship between Technical High School Brazilian students' Working Memory Capacity (WMC), inference generation and reading comprehension in L2. A group of 36 students from the third year of the Technical High School Course at Instituto Federal do Rio Grande do Sul (IFRS), Campus Sertão, participated in this study. Participants were pre-intermediate speakers of English as an L2. The instruments used in this study comprised a Reading Span Test (RST); two texts, being one narrative and the other expository; a Pause Protocol and two sets of comprehension questions (one for each text). Data from participants' Reading Span Test, the inferences they generated during reading (categorized in accordance with Narvaez; Broek; Ruiz' (1999) Inference Categorization Model), as well as their answers in the reading comprehension questions were analyzed both qualitatively and quantitatively, and the main results show that WMC positively correlates with reading comprehension, and also with explanatory inferences, which are strictly connected to reading comprehension.

- KEYWORDS: reading comprehension; working memory capacity; inference generation.

\section{REFERÊNCIAS}

ALPTEKIN, C.; ERÇETIN, G. The role of L1 and L2 working memory in literal and inferential comprehension in L2 reading. Journal of Research in Reading, Leeds, v.33, n.2, 2010.

ASHCRAFT, M. H. Human memory and cognition. New York: Harper Collins, 1994.

BADDELEY, A. D. The episodic buffer: a new component of working memory? Trends in Cognitive Sciences, Oxford, v.4, p.417-423, 2000.

BADDELEY, A. Working memory. Science, [s. l.], v.255, p.556-559, 1992.

BADDELEY, A. Human memory: theory and practice. Hove: Lawrence Erlbaum, 1990.

BADDELEY, A. D.; HITCH, G. Working memory. In: BOWER, G. H. (ed.). The psychology of Learning and Motivation. New York: Academic Press, 1974. v.8, p.47-89. 
BADDELEY, A. D.; HITCH, G. J. Developments in the concept of working memory. Neuropsychology, Philadelphia, v.8, n.4, p.485-493, 1994. Disponível em: https://doi. org/10.1037/0894-4105.8.4.485. Acesso em: 13 jan. 2022.

BADDELEY, A. D.; LOGIE, R. The multiple component model. In: MIYAKE, A.; SHAH, P. (ed.). Models of working memory: mechanisms of active maintenance and executive control. Ney York: Cambridge University Press, 1999. p.28-61.

BAILER, C. Working memory capacity and attention to form and meaning in EFL reading. 2011. Dissertation (Master in English) — Universidade Federal de Santa Catarina, Santa Catarina, 2011.

BARETTA, L. The process of inference making in reading comprehension: an ERP analysis. 2008. Thesis (Doctor in English) — Universidade Federal de Santa Catarina, Santa Catarina, 2008.

BERGSLEITHNER, J. Working memory capacity and L2 writing performance. Ciências \& Cognição, [s. l.], v.15, n.2, p.2-20, 2010.

BRITTON B. K. et al. Use of cognitive capacity in reading: effects of some content features of text. Discourse Processes, Norwood, v.6, p.39-57, 1983.

BUDD, D.; WHITNEY, P.; TURLEY, K. Individual differences in working memory strategies for reading expository text. Memory \& Cognition, Austin, v.23, n.6, p.735-748, 1995.

BROEK, P. van den; LORCH, R. F. Network representations of causal relations in memory for narrative texts: evidence from primed recognition. Discourse Processes, Norwood, v.16, n.1-2, p.75-98, 1993. Disponível em: https://doi. org/10.1080/01638539309544830. Acesso em: 13 jan. 2022.

BROEK, P. van den; RISDEN, K.; E. H. The role of readers' standards for coherence in the generation of inferences during reading. In: LORCH, R. F.; O'BRIEN, E. (ed.). Sources of coherence in reading. New Jersey: Lawrence Erlbaum Associates, 1995. p.353-373.

BROEK, P. van den; ROHLEDER, L.; NARVAEZ, D. Cognitive processes in the comprehension of literary texts. In: OOSTENDORP, H.; ZWAAN, R. (ed.). Naturalistic Text Comprehension. New Jersey: Ablex, 1994. p.229-246.

CALDART, D. The effect of genre expectation on EFL Brazilian students' inference generation and reading comprehension. 2012. Dissertation (Master in English) Universidade Federal de Santa Catarina, Florianópolis, 2012.

CANTOR, J.; ENGLE, R. Working memory capacity as long-term memory activation: an individual-differences approach. Journal of Experimental Psychology: Learning, Memory and Cognition, Arlington, v.19, n.5, p.1101-1114, 1993. 
CAVALCANTI, M. Interação leitor-texto: aspectos de interpretação pragmática. Campinas: UNICAMP, 1989.

DANEMAN, M.; CARPENTER, P. Individual differences in integrating information between and within sentences. Journal of experimental psychology: learning, memory and cognition, Arlington, v.9, n.4, p.561-584, 1983.

DANEMAN, M.; CARPENTER, P. Individual differences in working memory and reading. Journal of Verbal Learning and Verbal Behavior, New York, v.19, p.450-466, 1980.

DAVIES, F. Introducing reading. England: Penguin Books, 1995.

DIJK, T. van; KINTSCH, W. Strategies of discourse comprehension. New York: Academic Press, 1983.

ERICSSON, K. A.; SIMON, H.A. Protocol analysis: verbal reports as data. Cambridge: MIT Press, 1993.

ESKEY, D. Holding in the bottom: an interactive approach to the language problems of second language readers. In: CARRELL, P. L.; DEVINE J.; ESKEY, D. (ed.). Interactive Approaches to Second Language Reading. New York: Cambridge University Press, 1998. p.93-100.

FINARDI, K.; WEISSHEIMER, J. On the relationship between working memory capacity and L2 speech development. Signótica, Goiânia, v.20, p.367-391, 2008.

GAGNÉ, E. D.; YEKOVICH, C. W.; YEKOVICH, F. R. The cognitive psychology of school learning. New York: Harper Collins, 1993.

GERBER, R. M.; TOMITCH, L. M. B. Leitura e cognição: propósitos de leitura diferentes influem na geração de inferências? Revista Acta Scieniarum, Maringá, v.30, n.2, p.139-147, 2008.

GRAESSER, A. C. Prose comprehension beyond the word. New York: SpringerVerlag, 1981.

GRAESSER, A. C.; KREUZ, R. J. A theory of inference generation during text comprehension. Discourse Processes, Norwood, v.16, p.3-34, 1993.

GRAESSER, A. C.; SINGER, M.; TRABASSO, T. Constructing inferences during narrative text comprehension. Psychological Review, Washington, v.101, n.3, p.371$395,1994$.

HORIBA, Y. Reader control in reading: effects of language competence, text type and task. Discourse processes, Norwood, v.29, n.3, p.223-267, 2000.

JOH, J.; PLAKANS, L. Working memory in L2 reading comprehension: the influence of prior knowledge. System, [s. l.], p.1-14, 2017. 
JUFFS, A.; HARRINGTON, M. Aspects of working memory in L2 Learning. Language Teaching: Reviews and Studies, Cambridge, v.42, n.2, p.137-166, 2011.

JUST, M.; CARPENTER, P. A capacity theory of comprehension: individual differences in working memory. Psychological Review, Washington, v.99, p.122-149, 1992.

KRAAL, A. et al. Processing of expository and narrative texts by lowand highcomprehending children. Reading and Writing, Dordrecht, v.31, p.2017-2040, 2018.

KINTSCH, W.; DIJK, T. van. Toward a model of text comprehension and production. Psychological Review, Washington, v.85, n.5, p.363-394, 1978.

$\mathrm{KODA}, \mathrm{K}$. Insights into second language reading: a cross-linguistic approach. New York: Cambridge University Press, 2008.

LEESER, M. J. Learner-based factors in L2 reading comprehension and processing grammatical form: topic familiarity and working memory. Language Learning, Ann Arbor, v.57, p.229-270, 2007.

LINCK, J. A. et al. Working memory and second language comprehension and production: a meta-analysis. Psychonomic Bulletin and Review, Austin, v.21, p.861-883, 2013.

LINDERHOLM, T. Predictive inference generation as a function of working memory capacity and causal text constraints. Discourse Processes, Norwood, v.34, n.3, p.259-280, 2002.

LINDERHOLM, T.; BROEK, P. van den. The effect of reading purpose and working memory capacity on the processing of expository text. Journal of Educational Psychology, Airlington, v.94, n.4, p.778-784, 2002.

LORCH, R. F.; LORCH, E. P.; KLUSEWITZ, M. A. College students' conditional knowledge about reading. Journal of Educational Psychology, Airlington, v.85, p.239-252, 1993.

MAGLIANO, J.; GRAESSER, A.; TRABASSO, T. Strategic Processing During Comprehension. Journal of Educational Psychology, Airlington, v.91, n.4, p.615-629, 1999.

MARQUES, A. Inglês série Brasil. São Paulo: Ática, 2005.

MASSON, M. ; MILLER, J. Working memory and individual differences in comprehension and memory of text. Journal of Educational Psychology, Airlington, v.75, n.2, p.314-318, 1983.

MCNAMARA, D.; SCOTT, J. Working memory capacity and strategy use. Memory \& Cognition, Austin, v.29, n.1, p.10-17, 2001. 
NARVAEZ, D. Individual differences that influence reading comprehension. In: PRESSLEY, M. BLOOK, C. (ed.). Reading Comprehension Instruction. New York: Grilford, 2002. p.158-175.

NARVAEZ, D.; BROEK, P. van den; RUIZ, A. The influence of reading purpose on inference generation and comprehension in reading. Journal of Educational Psychology, Arlington, v.91, n.3, p.488-496, 1999.

PEARSON, P. D.; JOHNSON, D. D. Teaching reading comprehension. New York: Holt, Rinehart and Wiston, 1978.

PRESSLEY, M.; AFFLERBACH, P. Verbal protocols of reading: the nature of constructively responsive reading. Hillsdale: Lawrence Erlbaum, 1995.

ROSCIOLI, D. C. The relationship between technical high school Brazilian students' working memory capacity, pre-reading activities, and inference generation in reading comprehension in L2. Tese (Doutorado em Estudos da Linguagem) - Universidade Federal de Santa Catarina, Florianópolis, 2017.

RUMELHART, D. Schemata: the building blocks of cognition. In: GUTHRIE, J. (ed.). Comprehension and teaching: research reviews. Newark: International Reading Association, 1981. p.3-26.

RUMELHART, D. Toward an interactive model of reading. In: DOMINIC, S. I. (ed.). Attention and Performance IV. Hillsdale: Lawrence Erlbaum, 1977. p.573-603.

SAGARRA, N. Longitudinal effects of working memory on L2 grammar and reading abilities. Second Language Research, London, v.33, n.3, p.341-363, 2017.

SINGER, M. et al. Individual differences in bridging inference processes. Memory \& Cognition, Austin, v.20, n.5, p.539-548, 1992.

TOMITCH, L. M. B. Reading: text organization perception and working memory capacity. Florianópolis: PGI/UFSC, 2003. (ARES - Advanced Research in English Series).

TOMITCH, L. M. B. Schema activation and text comprehension. Fragmentos: Revista de Língua e Literatura Estrangeiras, Florianópolis, v.3, n.2, p.29-43, 1991.

TORRES, A. Working memory capacity and reader's performance on main idea construction in L1 and L2. 2003. Thesis (Doctor in English) — Universidade Federal de Santa Catarina, Florianópolis, 2003.

TRABASSO, T.; MAGLIANO, J. P. Conscious understanding during comprehension. Discourse Processes, Norwood, v.21, p.255-287, 1996. 
TRABASSO, T.; SUH, S. Understanding text: achieving explanatory coherence through on-line inferences and mental operations in working memory. Discourse processes, Norwood, v.16, n.1-2, p.3-34, 1993.

URQUHART, S.; WEIR, C. Reading in a second language: process, product and practice. New York: Longman, 1998.

WALTER, C. Transfer of reading comprehension skills to L2 is linked to mental representations of text and to L2 working memory. Applied Linguistics, Oxford, v.25, n.3, p.315-339, 2006.

WANG, W.; LIN, W. Recent studies into working memory capacity and L2 reading comprehension. Frontiers in Educational Research, Beijing, v.2, n.2, p.170-175, 2019.

WEISSHEIMER, J.; MOTA, M. Individual differences in working memory capacity and the development of L2 speech production. Issues in Applied Linguistics, Oakland, v.17, n.2, p.93-112, 2009.

WHITNEY, P.; RITCHIE, B. G.; CLARK, M. B. Working memory capacity and the use of elaborative inferences in text comprehension. Discourse Processes, Norwood, v.14, p.133-145, 1991.

YEARI, M. The role of working memory in inference generation during reading comprehension: retention, (re)activation, or suppression of verbal information? Learning and Individual Differences, Oxford, v.56, p.1-12, 2017.

YEARI, M.; BROEK, P. van den. The role of textual semantic constraints in knowledgebased inference generation during reading comprehension: a computational approach. Memory, [s. l.], v.23, n.8, p.1193-1214, 2015.

ZWAAN, R.; BROWN, C. The Influence of language proficiency and comprehension skill on situation-model construction. Discourse Processes, Norwood, v.21, p.289-327, 1996.

Received on April 6, 2020

Approved on September 24, 2020 